\title{
Convex meromorphic mappings
}

\author{
by Albert E. Livingston (Newark, Del.)
}

\begin{abstract}
We study functions $f(z)$ which are meromorphic and univalent in the unit disk with a simple pole at $z=p, 0<p<1$, and which map the unit disk onto a domain whose complement is either convex or is starlike with respect to a point $w_{0} \neq 0$.
\end{abstract}

1. Introduction. Let $S(p), 0<p<1$, be the class of functions meromorphic and univalent in the unit disk $\Delta=\{z:|z|<1\}$ with a simple pole at $z=p$ with a power series expansion $f(z)=z+b_{2} z^{2}+\ldots$ for $|z|<p$. The class $S(p)$ has been investigated by a number of authors. We let $C(p)$ be the subclass of $S(p)$ made up of functions $f$ such that $\overline{\mathbb{C}} \backslash f[\Delta]$ is a convex set. Royster [11] considered the class $K(p)$ consisting of members of $S(p)$ for which there exists $\delta, 0<\delta<1$, so that for $\delta<|z|<1$,

$$
\operatorname{Re}\left[1+\frac{z f^{\prime \prime}(z)}{f^{\prime}(z)}\right]<0 .
$$

Obviously $K(p) \subset C(p)$. Royster also studied the class $\Sigma(p) \subset S(p)$ consisting of functions $f$ such that

$$
\operatorname{Re}\left[\frac{1+p z}{1-p z}-\frac{z+p}{1+p z}-\left(1+\frac{z f^{\prime \prime}(z)}{f^{\prime}(z)}\right)\right]>0
$$

for $z \in \Delta$, and proved that $K(p)=\Sigma(p)$ for $0<p<2-\sqrt{3}$ but for $p>2-\sqrt{3}, K(p)$ is a proper subset of $\Sigma(p)$. Pfaltzgraff and Pinchuk [10] essentially proved that $C(p)=\Sigma(p)$ for $0<p<1$, by way of the Herglotz representation of functions of positive real part [12]. We will give another proof of this fact. We will also consider several coefficient problems. If $f$ is a member of $S(p)$ we will consider the two expansions

$$
f(z)=z+\sum_{n=2}^{\infty} b_{n} z^{n}, \quad|z|<p,
$$

1991 Mathematics Subject Classification: Primary 30C45.

Key words and phrases: convex, starlike, meromorphic. 
and

$$
f(z)=\sum_{n=-1}^{\infty} a_{n}(z-p)^{n}, \quad|z-p|<1-p .
$$

Goodman [2] conjectured that if $f$ is a member of $S(p)$, then

$$
\left|b_{n}\right| \leq \frac{1+p^{2}+\ldots+p^{2 n-2}}{p^{n-1}} .
$$

Jenkins [3] proved that (1.3) is true for any value of $n$ for which the Bieberbach conjecture holds. Since DeBrange [1] has now proven that conjecture to be valid for all $n$, it follows that (1.3) holds for all $n$. The inequality (1.3) is actually sharp in $C(p)$, since the extremal function $f(z)=$ $-p z /(z-p)(1-p z)$ maps $\Delta$ onto the complement of the real interval $\left[-p /(1-p)^{2},-p /(1+p)^{2}\right]$. Miller [9] proved that if $f$ is a member of $\Sigma(p)$, then

from which it follows that

$$
\left|b_{2}-\frac{\left(1+p^{2}+p^{4}\right)}{p\left(1+p^{2}\right)}\right| \leq \frac{p}{1+p^{2}}
$$

$$
\operatorname{Re}\left(b_{2}\right) \geq \frac{1+p^{4}}{p\left(1+p^{2}\right)}>1 .
$$

Miller [9] also obtained a lower bound for $\operatorname{Re} b_{3}$, which is positive for $p$ near 0 , for $f$ in $C(p)=\Sigma(p)$. We will obtain the sharp inequality

$$
\operatorname{Re} b_{3} \geq \frac{1-p^{2}+p^{4}}{p^{2}}>1
$$

if $f$ is in $C(p)=\Sigma(p)$.

Concerning the expansion (1.2), the sharp estimate $\left|a_{-1}\right| \leq p^{2} /\left(1-p^{2}\right)$ if $f$ is a member of $S(p)$ has been proven by Kirwan and Schober [4] and also Komatu [5]. Komatu [5] obtained the sharp bound on $\left|a_{1}\right|$ for $f$ in $S(p)$ and the extremal function is a member of $C(p)$. We will give another proof in $C(p)$ and also obtain the sharp bound on $\left|a_{2}\right|$ for $f$ in $C(p)$.

2. The class of $C(p)$. In this section we will give a different necessary and sufficient condition for membership in $C(p)$ and a new proof that $C(p)=$ $\Sigma(p)$.

THEOREM 1. $f$ is a member of $C(p)$ if and only if for $z \in \Delta$,

$$
\operatorname{Re}\left[1+p^{2}-2 p z+\frac{(z-p)(1-p z) f^{\prime \prime}(z)}{f^{\prime}(z)}\right]<0 .
$$

Proof. If $f$ is a member of $S(p)$ let $h(z)=f((z+p) /(1+p z))$; then $h$ has a simple pole at $z=0$ and $\overline{\mathbb{C}} \backslash h[\Delta]=\overline{\mathbb{C}} \backslash f[\Delta]$. Thus, $f$ is a member of 
$C(p)$ if and only if $h$ is convex with a simple pole at $z=0$. This is the case if and only if [10]

$$
\operatorname{Re}\left(1+\frac{z h^{\prime \prime}(z)}{h^{\prime}(z)}\right)<0
$$

for $z \in \Delta$. A straightforward computation gives

$$
\operatorname{Re}\left(1+\frac{z h^{\prime \prime}(z)}{h^{\prime}(z)}\right)=\operatorname{Re} Q(z)
$$

where

$$
Q(z)=\frac{1-p z}{1+p z}+\frac{\frac{\left(1-p^{2}\right) z}{(1+p z)^{2}} f^{\prime \prime}\left(\frac{z+p}{1+p z}\right)}{f^{\prime}\left(\frac{z+p}{1+p z}\right)} .
$$

But $\operatorname{Re} Q(z)<0$ for $z \in \Delta$ if and only if $\operatorname{Re} Q((z-p) /(1-p z))<0$ for $z \in \Delta$. However,

$$
Q\left(\frac{z-p}{1-p z}\right)=\frac{1-p^{2}-2 p z}{\left(1-p^{2}\right)}+\frac{(z-p)(1-p z) f^{\prime \prime}(z)}{\left(1-p^{2}\right) f^{\prime}(z)},
$$

which gives $(2.1)$.

Remark. If $f$ is a member of $C(p)$ and

$$
P(z)=2 p z-1-p^{2}-\frac{(z-p)(1-p z) f^{\prime \prime}(z)}{f^{\prime}(z)}
$$

then $\operatorname{Re} P(z)>0, z \in \Delta, P(p)=1-p^{2}$ and $P^{\prime}(p)=0$.

Lemma 1. Let $P(z)$ satisfy $\operatorname{Re} P(z)>0, z \in \Delta$, and $P(0)=1$. If $0<p<1$, then for $z \in \Delta$,

$$
\operatorname{Re}\left[\frac{(z-p)(1-p z) P(z)+p}{z}-p z\right]>0 .
$$

Proof. Let $0<r<1$ and $P_{r}(z)=P(r z)$. Then

$$
Q_{r}(z)=\frac{(z-p)(1-p z) P_{r}(z)+p}{z}-p z
$$

is analytic for $|z| \leq 1$. If $|z|=1$, then

$$
Q_{r}(z)=\frac{(z-p)(1-p z) P_{r}(z)}{z}-p\left(z-\frac{1}{z}\right)
$$

and

$$
\operatorname{Re} Q_{r}(z)=|1-p z|^{2} \operatorname{Re} P_{r}(z)>0 .
$$


Since $Q_{r}(z)$ is analytic for $|z| \leq 1, \operatorname{Re} Q_{r}(z)>0$ for $z \in \Delta$. Letting $r \rightarrow 1$, we obtain for $z \in \Delta$,

$$
\operatorname{Re}\left[\frac{(z-p)(1-p z) P(z)+p}{z}-p z\right] \geq 0 .
$$

But equality cannot occur in the last inequality since the quantity on the left side equals $1-p^{2}$ when $z=p$.

Lemma 2. If $\operatorname{Re} P(z)>0$ for $z \in \Delta$ and $P(p)=1-p^{2}$, then for $z \in \Delta$,

$$
\operatorname{Re}\left[\frac{z P(z)-p+p z^{2}}{(z-p)(1-p z)}\right]>0 .
$$

Proof. Let $p<r<1$ and $\alpha=(r-1) p /\left(r-p^{2}\right)$ and $L_{r}(z)=r(z-$ $\alpha) /(1-\bar{\alpha} z)$. It is easily verified that $L_{r}[\Delta]=\{z:|z|<r\}$ and $L_{r}(p)=p$.

Let

$$
Q_{r}(z)=\frac{z P\left(L_{r}(z)\right)-p+p z^{2}}{(z-p)(1-p z)}
$$

$Q_{r}(z)$ is analytic for $|z| \leq 1$ and $\operatorname{Re} P\left(L_{r}(z)\right)>0$ for $|z| \leq 1$. If $|z|=1$ then

$$
\begin{aligned}
\operatorname{Re} Q_{r}(z) & =\operatorname{Re}\left[\frac{z P\left(L_{r}(z)\right)}{(z-p)(1-p z)}+\frac{p z(z-1 / z)}{(z-p)(1-p z)}\right] \\
& =\frac{1}{|1-p z|^{2}} \operatorname{Re} P\left(L_{r}(z)\right)>0 .
\end{aligned}
$$

Since $Q_{r}$ is analytic for $|z| \leq 1$, it follows that $\operatorname{Re} Q_{r}(z)>0$ for $z \in \Delta$. Letting $r \rightarrow 1$, we obtain for $z \in \Delta$,

$$
\operatorname{Re}\left[\frac{z P(z)-p+p z^{2}}{(z-p)(1-p z)}\right] \geq 0 .
$$

But equality cannot occur in the last inequality since the expression on the left equals 1 when $z=0$.

THEOREM 2. $C(p)=\Sigma(p)$ for $0<p<1$.

Proof. Let $f$ be a member of $\Sigma(p)$ and

$$
P(z)=-1-\frac{z f^{\prime \prime}(z)}{f^{\prime}(z)}+\frac{1+p z}{1-p z}-\frac{z+p}{z-p} .
$$

Then $\operatorname{Re} P(z)>0, z \in \Delta$, and $P(0)=1$. Straightforward computations give

$$
2 p z-1-p^{2}-\frac{(z-p)(1-p z) f^{\prime \prime}(z)}{f^{\prime}(z)}=\frac{(z-p)(1-p z) P(z)+p}{z}-p z .
$$

Therefore, by Lemma 1 ,

$$
\operatorname{Re}\left[2 p z-1-p^{2}-\frac{(z-p)(1-p z) f^{\prime \prime}(z)}{f^{\prime}(z)}\right]>0
$$

for $z \in \Delta$, and thus by Theorem $1, f$ is a member of $C(p)$. 
Conversely, suppose $f$ is a member of $C(p)$ and let

$$
P(z)=2 p z-1-p^{2}-\frac{(z-p)(1-p z) f^{\prime \prime}(z)}{f^{\prime}(z)} .
$$

Then by Theorem $1, \operatorname{Re} P(z)>0, z \in \Delta$, and $P(p)=1-p^{2}$. Straightforward computations give

$$
-1-\frac{z f^{\prime \prime}(z)}{f^{\prime}(z)}-\frac{z+p}{z-p}+\frac{1+p z}{1-p z}=\frac{z P(z)-p+p z^{2}}{(z-p)(1-p z)} .
$$

Thus, by Lemma 2,

$$
\operatorname{Re}\left[-1-\frac{z f^{\prime \prime}(z)}{f^{\prime}(z)}-\frac{z+p}{z-p}+\frac{1+p z}{1-p z}\right]>0
$$

for $z \in \Delta$. Therefore $f$ is a member of $\Sigma(p)$.

3. The coefficients $a_{n}$. In this section we use Theorem 1 to study the coefficients $a_{1}$ and $a_{2}$ in (1.2), if $f$ is a member of $C(p)$. We will make use of the following lemma.

LEMMA 3. Let $P(z)$ be analytic in $\Delta$ and satisfy $\operatorname{Re} P(z)>0, z \in \Delta$, $P(p)=1-p^{2}$ and $P^{\prime}(p)=0,0<p<1$. If $P(z)=\left(1-p^{2}\right)+d_{2}(z-p)^{2}+$ $d_{3}(z-p)^{3}+\ldots$ for $|z-p|<1-p$, then

$$
\begin{aligned}
\left|d_{2}\right| & \leq \frac{2}{1-p^{2}}, \\
\left|\frac{p}{1-p^{2}} d_{2}+d_{3}\right| & \leq \frac{6 p}{\left(1-p^{2}\right)^{2}}, \quad 2 / 3 \leq p<1, \\
\left|\frac{p}{1-p^{2}} d_{2}+d_{3}\right| & \leq \frac{2\left(1+\frac{9}{4} p^{2}\right)}{1-p^{2}}, \quad 0<p \leq 2 / 3 .
\end{aligned}
$$

All the inequalities are sharp.

Proof. Let

$$
w(z)=\frac{P(z)-\left(1-p^{2}\right)}{P(z)+1-p^{2}} .
$$

Then $w(p)=0$ and $|w(z)| \leq 1, z \in \Delta$. Also

$$
w^{\prime}(z)=\frac{2\left(1-p^{2}\right) P^{\prime}(z)}{\left[P(z)+(1-p)^{2}\right]^{2}}
$$

and hence $w^{\prime}(p)=0$. Comparing coefficients in the expansions of both sides of

$$
\left[P(z)+\left(1-p^{2}\right)\right] w(z)=P(z)-\left(1-p^{2}\right)
$$

we obtain

$$
d_{2}=\left(1-p^{2}\right) w^{\prime \prime}(p)
$$


and

$$
\frac{p}{1-p^{2}} d_{2}+d_{3}=p w^{\prime \prime}(p)+(1-p) \frac{w^{\prime \prime \prime}(p)}{3} .
$$

We can write

$$
w(z)=\phi\left(\frac{z-p}{1-p z}\right)
$$

where $\phi$ is analytic for $|z|<1, \phi(0)=\phi^{\prime}(0)=0$ and $|\phi(z)| \leq 1, z \in \Delta$. In particular, we obtain

$$
w^{\prime \prime}(p)=\frac{\phi^{\prime \prime}(0)}{\left(1-p^{2}\right)^{2}} .
$$

Since $\left|\phi^{\prime \prime}(0) / 2\right| \leq 1$, we have $\left|w^{\prime \prime}(p)\right| \leq 2 /\left(1-p^{2}\right)^{2}$. Thus from (3.4) we obtain

which is $(3.1)$.

$$
\left|d_{2}\right|=\left(1-p^{2}\right)\left|w^{\prime \prime}(p)\right| \leq \frac{2}{1-p^{2}},
$$

Next from (3.5) we obtain

$$
\frac{p}{1-p^{2}} d_{2}+d_{3}=\frac{1}{\left(1-p^{2}\right)^{2}}\left[\frac{\phi^{\prime \prime \prime}(0)}{3}+3 p \phi^{\prime \prime}(0)\right] .
$$

If $\phi(z)=c_{2} z^{2}+c_{3} z^{3}+\ldots, z \in \Delta$, then

$$
\frac{p}{1-p^{2}} d_{2}+d_{3}=\frac{2}{\left(1-p^{2}\right)^{2}}\left[c_{3}+3 p c_{2}\right] .
$$

Using known inequalities for bounded functions, we obtain

$$
\left|c_{3}+3 p c_{2}\right| \leq\left|c_{3}\right|+3 p\left|c_{2}\right| \leq 1-\left|c_{2}\right|^{2}+3 p\left|c_{2}\right| .
$$

Therefore

$$
\left|\frac{p}{1-p^{2}} d_{2}+d_{3}\right| \leq \frac{2}{\left(1-p^{2}\right)^{2}}\left[1+3 p\left|c_{2}\right|-\left|c_{2}\right|^{2}\right] .
$$

Let $x=\left|c_{2}\right|$ and $h(x)=1+3 p x-x^{2}, 0 \leq x \leq 1$. Then $h^{\prime}(x)=3 p-2 x$. If $p \geq 2 / 3$, then $h^{\prime}(x) \geq 0$ for $0 \leq x \leq 1$ and hence

$$
h(x) \leq h(1)=3 p, \quad 2 / 3 \leq p<1 .
$$

If $0<p<2 / 3$, then $h(x)$ achieves its maximum at $x=3 p / 2$. Hence

$$
h(x) \leq 1+\frac{9}{4} p^{2}, \quad 0<p \leq 2 / 3 .
$$

Combining (3.6), (3.7) and (3.8) gives (3.2) and (3.3).

Equality is attained in (3.1) by the function

$$
P(z)=\frac{1+p^{2}-4 p z+\left(1+p^{2}\right) z^{2}}{1-z^{2}},
$$

which is obtained by taking $w(z)=[(z-p) /(1-p z)]^{2}$. The same function gives equality in (3.2). 
If $0<p<2 / 3$, let

$$
\phi(z)=\frac{z^{2}\left(z+\frac{3}{2} p\right)}{1+\frac{3}{2} p z}
$$

and $w(z)=\phi((z-p) /(1-p z))$. The resulting function $P(z)=\left(1-p^{2}\right)(1+$ $w(z)) /(1-w(z))$ gives equality in (3.3).

TheOREM 3. Let $f$ be a member of $C(p)$ and have the expansion (1.2). Then

$$
\begin{aligned}
& \left|a_{1}\right| \leq \frac{p^{2}}{\left(1-p^{2}\right)^{3}}, \\
& \left|a_{2}\right| \leq \frac{\left(4+9 p^{2}\right)\left|a_{-1}\right|}{12\left(1-p^{2}\right)^{3}}, \quad 0<p \leq 2 / 3, \\
& \left|a_{2}\right| \leq \frac{p}{\left(1-p^{2}\right)^{3}}\left|a_{-1}\right| \leq \frac{p^{3}}{\left(1-p^{2}\right)^{4}}, \quad 2 / 3 \leq p \leq 1 .
\end{aligned}
$$

All the inequalities are sharp.

R e m a rk. Making use of the area theorem, Komatu [5] proved inequality (3.9) for the larger class $S(p)$.

Proof of Theorem 3. Let

$$
P(z)=2 p z-1-p^{2}-\frac{(z-p)(1-p z) f^{\prime \prime}(z)}{f^{\prime}(z)} .
$$

Then $P(z)$ satisfies the hypotheses of Lemma 3. Comparing coefficients on both sides of the equation

$$
\left[2 p(z-p)-\left(1-p^{2}\right)\right] f^{\prime}(z)-(z-p)\left[\left(1-p^{2}\right)-p(z-p)\right] f^{\prime \prime}(z)=P(z) f^{\prime}(z)
$$

we obtain

$$
2 a_{1}\left(1-p^{2}\right)=a_{-1} d_{2}
$$

and

$$
6\left(1-p^{2}\right) a_{2}=2 p a_{1}+a_{-1} d_{3} .
$$

Combining (3.1) and (3.12) gives

$$
\left|a_{1}\right| \leq \frac{\left|a_{-1}\right|}{\left(1-p^{2}\right)^{2}} .
$$

However, $\left|a_{-1}\right| \leq p^{2} /\left(1-p^{2}\right)$ (cf. [4], [5]), giving (3.9).

Combining (3.12) and (3.13) gives

$$
a_{2}=\frac{1}{6\left(1-p^{2}\right)}\left[\frac{p}{1-p^{2}} d_{2}+d_{3}\right] a_{-1} .
$$

If $0<p \leq 2 / 3$, then (3.3) and (3.14) gives (3.10). If $2 / 3 \leq p<1$, then (3.2) combined with (3.14) gives (3.11). 
Equality is attained in (3.9) and (3.11) by $f(z)=-p z /((z-p)(1-p z))$. If $0<p \leq 2 / 3$, equality is attained in (3.10) by the function $f$ which satisfies

$$
2 p z-1-p^{2}-\frac{(z-p)(1-p z) f^{\prime \prime}(z)}{f^{\prime}(z)}=P(z)
$$

where $P(z)$ is the function satisfying the hypotheses of Lemma 3 and giving equality in (3.3). Since $\operatorname{Re} P(z)=0$ on $|z|=1$ with finitely many exceptions and since

$$
1+\frac{z f^{\prime \prime}(z)}{f^{\prime}(z)}=\frac{z}{(z-p)(1-p z)}\left[p\left(z-\frac{1}{z}\right)-P(z)\right]
$$

it follows that on $|z|=1$,

$$
\operatorname{Re}\left(1+\frac{z f^{\prime \prime}(z)}{f^{\prime}(z)}\right)=\frac{1}{|1-p z|^{2}} \operatorname{Re} P(z)=0
$$

with finitely many exceptions.

Laborious computations give

$$
P(z)=\frac{\left(1-p^{2}\right)\left(2+2 p-p^{2}\right)}{2-2 p-p^{2}} \cdot \frac{(1+z)\left(z-e^{i \gamma}\right)\left(z-e^{-i \gamma}\right)}{(1-z)\left(z-e^{i \beta}\right)\left(z-e^{-i \beta}\right)}
$$

where $e^{i \gamma} \neq e^{i \beta}$, and $e^{i \beta}$ is not real for $0<p<2 / 3$. Thus $\operatorname{Re}(1+$ $\left.z f^{\prime \prime}(z) / f^{\prime}(z)\right)=0$ on $|z|=1$ with 3 exceptional points. It follows that for the extremal function in the case $0<p<2 / 3, \overline{\mathbb{C}} \backslash f[\Delta]$ is the interior of a triangle.

Re mark. In the case $0<p<2 / 3$ of Theorem 3 , using the inequality $\left|a_{-1}\right| \leq p^{2} /\left(1-p^{2}\right)$ in $(3.10)$ does not result in a sharp inequality.

THEOREM 4. If $f$ is a member of $C(p)$ with expansion (1.2), then

$$
\left|p+\frac{a_{0}\left(1-p^{2}\right)}{a_{-1}}\right| \leq \frac{1+p^{2}}{p},
$$

and the inequality is sharp.

Proof. Let

$$
h(z)=\frac{-a_{-1}}{\left(1-p^{2}\right) f\left(\frac{p-z}{1-p z}\right)},
$$

then $h$ is a member of $S(p)$ and for $|z-p|<1-p$,

$$
h(z)=z+\left(p+\frac{\left(1-p^{2}\right) a_{0}}{a_{-1}}\right) z^{2}+\ldots
$$

Using (1.3) when $n=2$, we get

$$
\left|p+\frac{\left(1-p^{2}\right) a_{0}}{a_{-1}}\right| \leq \frac{1+p^{2}}{p} .
$$

Equality is attained by $f(z)=-p z /((z-p)(1-p z))$. 
4. The coefficients $b_{n}$. Let $f$ be a member of $C(p)$ and have the expansion (1.1) for $|z|<p$. As remarked in the introduction, sharp upper bounds on $\left|b_{n}\right|$ are known for all $n$ and a sharp lower bound on $\operatorname{Re}\left(b_{2}\right)$ follows from results in [9]. In this section we will obtain a sharp lower bound on $\operatorname{Re}\left(b_{3}\right)$ which suggests a conjecture concerning $\operatorname{Re}\left(b_{n}\right)$ for all $n$.

TheOREM 5. Let $f$ be a member of $C(p)$ with expansion (1.1). Then

$$
\operatorname{Re} b_{2} \geq \frac{1+p^{4}}{p\left(1+p^{2}\right)}>1
$$

and

$$
\operatorname{Re} b_{3} \geq \frac{1-p^{2}+p^{4}}{p^{2}}=\frac{1+p^{6}}{p^{2}\left(1+p^{2}\right)}>1 .
$$

Both inequalities are sharp, each being attained by the function

$$
f(z)=\frac{p\left(1+p^{2}\right) z-2 p^{2} z^{2}}{\left(1-p^{2}\right)(p-z)(1-p z)} .
$$

Proof. Let

$$
P(z)=2 p z-1-p^{2}-\frac{\left(-p+\left(1+p^{2}\right) z-p z^{2}\right) f^{\prime \prime}(z)}{f^{\prime}(z)},
$$

then $\operatorname{Re} P(z)>0, z \in \Delta, P(p)=1-p^{2}$ and $P^{\prime}(p)=0$. Let $P(z)=$ $c_{0}+c_{1} z+c_{2} z^{2}+\ldots$ Comparing coefficients on both sides of the equation

$$
P(z) f^{\prime}(z)=\left[2 p z-\left(1+p^{2}\right)\right] f^{\prime}(z)-\left[-p+\left(1+p^{2}\right) z-p z^{2}\right] f^{\prime \prime}(z),
$$

we obtain

$$
c_{0}=2 p b_{2}-\left(1+p^{2}\right)
$$

and

$$
2 c_{0} b_{2}+c_{1}=2 p-4\left(1+p^{2}\right) b_{2}+6 p b_{3} .
$$

Using (4.3) and (4.4) we obtain

$$
b_{2}=\frac{c_{0}+\left(1+p^{2}\right)}{2 p}
$$

and

$$
6 p^{2} b_{3}=c_{0}^{2}+3\left(1+p^{2}\right) c_{0}+p c_{1}+2\left(1+p^{2}+p^{4}\right) .
$$

Let $w(z)=\left[P(z)-\left(1-p^{2}\right)\right] /\left[P(z)+\left(1-p^{2}\right)\right]$, then $|w(z)|<1$ for $z \in \Delta$ and $w(p)=w^{\prime}(p)=0$. Thus we can write

$$
w(z)=\left(\frac{z-p}{1-p z}\right)^{2} \phi(z)
$$


where $|\phi(z)|<1$ for $z \in \Delta$. We have

$$
P(z)=\frac{\left(1-p^{2}\right)(1+w(z))}{1-w(z)} .
$$

Thus

$$
c_{0}=P(0)=\frac{\left(1-p^{2}\right)(1+w(0))}{1-w(0)}=\frac{\left(1-p^{2}\right)\left(1+p^{2} \phi(0)\right)}{1-p^{2} \phi(0)} .
$$

It follows that

$$
\operatorname{Re} c_{0} \geq\left(1-p^{2}\right) \frac{1-p^{2}|\phi(0)|}{1+p^{2}|\phi(0)|} \geq \frac{\left(1-p^{2}\right)^{2}}{1+p^{2}} .
$$

Using this inequality in conjunction with (4.5) gives (4.1), which has also been proven by Miller [9].

Next, we have

$$
\begin{aligned}
c_{1}=P^{\prime}(0) & =\frac{2\left(1-p^{2}\right) w^{\prime}(0)}{(1-w(0))^{2}} \\
& =\frac{2\left(1-p^{2}\right)\left[-2 p\left(1-p^{2}\right) \phi(0)+p^{2} \phi^{\prime}(0)\right]}{\left(1-p^{2} \phi(0)\right)^{2}} .
\end{aligned}
$$

Combining (4.6), (4.7) and (4.8), we eventually obtain

$$
\begin{aligned}
6 p^{2} b_{3}=\left(1-p^{2}\right)\left[\left(1-p^{2}\right)+\frac{2 p^{3} \phi^{\prime}(0)}{\left(1-p^{2} \phi(0)\right)^{2}}+3\left(1+p^{2}\right) \frac{1+p^{2} \phi(0)}{1-p^{2} \phi(0)}\right] \\
+2\left(1+p^{2}+p^{4}\right)
\end{aligned}
$$

Now let

$$
Q(z)=\frac{1+p^{2} \phi(z)}{1-p^{2} \phi(z)} .
$$

Then $\operatorname{Re} Q(z) \geq\left(1-p^{2}\right) /\left(1+p^{2}\right)>0$ for $z \in \Delta$ and (4.9) can be written as (4.10) $6 p^{2} b_{3}=\left(1-p^{2}\right)\left[\left(1-p^{2}\right)+p Q^{\prime}(0)+3\left(1+p^{2}\right) Q(0)\right]$

$$
+2\left(1+p^{2}+p^{4}\right) \text {. }
$$

Let $T(z)=Q(z)-\left(1-p^{2}\right) /\left(1+p^{2}\right)$. Since $\operatorname{Re} T(z)>0$ for $z \in \Delta$, it is known that

$$
\left|T^{\prime}(0)\right| \leq 2 \operatorname{Re} T(0) .
$$

Thus

$$
\left|Q^{\prime}(0)\right| \leq 2 \operatorname{Re}\left[Q(0)-\frac{1-p^{2}}{1+p^{2}}\right] .
$$

Hence

$$
2 \operatorname{Re} Q(0) \geq\left|Q^{\prime}(0)\right|+2 \frac{1-p^{2}}{1+p^{2}}
$$


Using the last inequality with (4.10) we obtain

$$
\begin{aligned}
6 p^{2} \operatorname{Re} b_{3} \geq & \left(1-p^{2}\right)\left[\left(1-p^{2}\right)-p\left|Q^{\prime}(0)\right|+\frac{3}{2}\left(1+p^{2}\right)\left|Q^{\prime}(0)\right|+3\left(1-p^{2}\right)\right] \\
& +2\left(1+p^{2}+p^{4}\right) \\
= & \left(1-p^{2}\right)\left[\left(1-p^{2}\right)+\frac{3+3 p^{2}-2 p}{2}\left|Q^{\prime}(0)\right|+3\left(1-p^{2}\right)\right] \\
& +2\left(1+p^{2}+p^{4}\right) \\
\geq & \left(1-p^{2}\right)\left[4\left(1-p^{2}\right)\right]+2\left(1+p^{2}+p^{4}\right) \\
= & 6\left(1-p^{2}+p^{4}\right),
\end{aligned}
$$

which gives (4.2).

An examination of the proof indicates that equality holds in (4.1) and (4.2) if and only if $\phi(z) \equiv-1$. This leads to the extremal function stated in the theorem.

R e mark. It seems reasonable to expect that the extremal function for Theorem 5 is extremal for all $n$. That is, we expect that if $f$ is a member of $C(p)$, then $\operatorname{Re}\left(b_{n}\right) \geq\left(1+p^{2 n}\right) /\left(p^{n-1}\left(1+p^{2}\right)\right)$ for all $n$.

5. Starlike functions. Miller [7]-[9] considered functions $f$ of $S(p)$ for which there exists $\varrho, 0<\varrho<1$, so that $\operatorname{Re}\left[z f^{\prime}(z) /\left(f(z)-w_{0}\right)\right]<0$ for $\varrho<|z|<1$ and a fixed $w_{0} \in \mathbb{C}, w_{0} \neq 0$. These functions map $\Delta$ onto the complement of a set which is starlike with respect to $w_{0}$. This class of functions is a subclass of the class $\Sigma^{*}\left(p, w_{0}\right)$ defined as the class of functions $f$ in $S(p)$ such that for $z \in \Delta$,

$$
\operatorname{Re}\left[\frac{p z}{1-p z}-\frac{p}{z-p}-\frac{z f^{\prime}(z)}{\left(f(z)-w_{0}\right)}\right]>0 .
$$

Actually, the two classes are the same if $0<p<\sqrt{3-2 \sqrt{2}}$ (cf. [9]). But for $p \geq \sqrt{3-2 \sqrt{2}}$ and proper choice of $w_{0}$ the first class is a proper subset of the second. We will prove that $\Sigma^{*}\left(p, w_{0}\right)$ is the class of all functions $f$ in $S(p)$ such that $\overline{\mathbb{C}} \backslash f[\Delta]$ is starlike with respect to $w_{0}$, which we denote by $\Sigma^{\mathrm{s}}\left(p, w_{0}\right)$.

THEOREM 6. $f$ is a member of $\Sigma^{\mathrm{s}}\left(p, w_{0}\right)$ if and only if, for $z \in \Delta$,

$$
\operatorname{Re}\left[\frac{(z-p)(1-p z) f^{\prime}(z)}{f(z)-w_{0}}\right]<0 .
$$

Proof. Suppose $f$ is a member of $S(p)$ and let

$$
g(z)=f\left(\frac{z+p}{1+p z}\right) \text {. }
$$


$f$ is a member of $\Sigma^{\mathrm{s}}\left(p, w_{0}\right)$ if and only if $\overline{\mathbb{C}} \backslash g(\Delta)$ is starlike with respect to $w_{0}$. This is the case if and only if $F(z)=g(z)-w_{0}$ maps $\Delta$ onto the complement of a set which is starlike with respect to the origin. Since $F$ has its pole at the origin, $\overline{\mathbb{C}} \backslash F[\Delta]$ is starlike with respect to the origin if and only if $\operatorname{Re}\left[z F^{\prime}(z) / F(z)\right]<0$ for $z \in \Delta$. The last inequality is true if and only if

$$
\operatorname{Re}\left[\left(\frac{z-p}{1-p z}\right) F^{\prime}\left(\frac{z-p}{1-p z}\right) / F\left(\frac{z-p}{1-p z}\right)\right]<0
$$

for $z \in \Delta$. A straightforward computation gives

$$
\left(\frac{z-p}{1-p z}\right) F^{\prime}\left(\frac{z-p}{1-p z}\right) / F\left(\frac{z-p}{1-p z}\right)=\frac{(z-p)(1-p z) f^{\prime}(z)}{\left(1-p^{2}\right)\left(f(z)-w_{0}\right)}
$$

and the theorem follows.

Theorem 7. $\Sigma^{\mathrm{S}}\left(p, w_{0}\right)=\Sigma^{*}\left(p, w_{0}\right)$ for all $p, 0<p<1$, and all $w_{0} \neq 0$.

Proof. Let $f$ be a member of $\Sigma^{*}\left(p, w_{0}\right)$ and

$$
P(z)=\frac{p z}{1-p z}-\frac{p}{z-p}-\frac{z f^{\prime}(z)}{f(z)-w_{0}},
$$

then $\operatorname{Re} P(z)>0$ for $z \in \Delta$ and $P(0)=1$. From this we obtain

$$
\frac{(z-p)(1-p z) f^{\prime}(z)}{f(z)-w_{0}}=-\frac{(z-p)(1-p z) P(z)+p\left(1-z^{2}\right)}{z} .
$$

Let $0<r<1$ and

$$
Q_{r}(z)=\frac{(z-p)(1-p z) P(r z)+p\left(1-z^{2}\right)}{z},
$$

then $Q_{r}(z)$ is analytic for $|z| \leq 1$, and

$$
\operatorname{Re} Q_{r}(z)=|1-p z|^{2} \operatorname{Re} P(r z)>0
$$

for $|z|=1$. Thus $\operatorname{Re} Q_{r}(z)>0$ for $z \in \Delta$. If we let $r \rightarrow 1$, we obtain

$$
\operatorname{Re}\left[\frac{(z-p)(1-p z) P(z)+p\left(1-z^{2}\right)}{z}\right] \geq 0 .
$$

However, the expression on the left side of (5.2) is strictly positive for $z=p$. Thus equality cannot occur in (5.2). Hence from (5.1),

$$
\operatorname{Re}\left[\frac{(z-p)(1-p z) f^{\prime}(z)}{f(z)-w_{0}}\right]<0
$$

for $z \in \Delta$. Thus by Theorem $6, f$ is a member of $\Sigma^{\mathrm{s}}\left(p, w_{0}\right)$.

Conversely, suppose $f$ is a member of $\Sigma^{\mathrm{s}}\left(p, w_{0}\right)$ and let

$$
P(z)=-\frac{(z-p)(1-p z) f^{\prime}(z)}{f(z)-w_{0}},
$$


then $\operatorname{Re} P(z)>0$ for $z \in \Delta$ and $P(p)=1-p^{2}$. We obtain

$$
\frac{p z}{1-p z}-\frac{p}{(z-p)}-\frac{z f^{\prime}(z)}{f(z)-w_{0}}=\frac{z P(z)-p\left(1-z^{2}\right)}{(z-p)(1-p z)} .
$$

By Lemma 2 the real part of the expression on the right side of (5.3) is strictly positive for $z$ in $\Delta$. Thus $f$ is a member of $\Sigma^{*}\left(p, w_{0}\right)$.

Miller [9] has given some estimates of coefficients in the expansion (1.1) if $f$ is a member of $\Sigma^{*}\left(p, w_{0}\right)=\Sigma^{\mathrm{s}}\left(p, w_{0}\right)$. We will next give sharp bounds on a few coefficients in the expansion (1.2).

THEOREM 8. If $f(z)$ is a member of $\Sigma^{*}\left(p, w_{0}\right)$ and has expansion (1.2) for $|z-p|<1-p$ then

$$
\left|a_{0}-w_{0}\right| \leq \frac{2+p}{1-p^{2}}\left|a_{-1}\right|
$$

and

$$
\left|a_{1}\right| \leq \frac{\left|a_{-1}\right|}{\left(1-p^{2}\right)^{2}}
$$

Both inequalities are sharp.

Pr o of. We first prove inequality (5.5). Let

$$
P(z)=\frac{-(z-p)(1-p z) f^{\prime}(z)}{f(z)-w_{0}},
$$

then $\operatorname{Re} P(z)>0$ for $z \in \Delta$ and $P(p)=1-p^{2}$. Let

$$
P(z)=\left(1-p^{2}\right)+\sum_{n=1}^{\infty} c_{n}(z-p)^{n}
$$

for $|z-p|<1-p$. Comparing coefficients on both sides of the equation

$$
\left(f(z)-w_{0}\right) P(z)=-(z-p)(1-p z) f^{\prime}(z),
$$

we obtain

$$
\begin{aligned}
a_{-1} c_{1}+\left(1-p^{2}\right)\left(a_{0}-w_{0}\right) & =-p a_{-1}, \\
a_{-1} c_{2}+\left(a_{0}-w_{0}\right) c_{1}+\left(1-p^{2}\right) a_{1} & =-a_{1}\left(1-p^{2}\right) .
\end{aligned}
$$

Combining (5.6) and (5.7), we eventually obtain

$$
-2\left(1-p^{2}\right)^{2} a_{1}=a_{-1}\left[\left(1-p^{2}\right) c_{2}-p c_{1}-c_{1}^{2}\right] .
$$

We now claim that $\left|\left(1-p^{2}\right) c_{2}-p c_{1}-c_{1}^{2}\right| \leq 2$. To prove this, let

$$
Q(z)=\frac{1}{1-p^{2}} P\left(\frac{z+p}{1+p z}\right)
$$


then $\operatorname{Re} P(z)>0$ for $z \in \Delta$ and $Q(0)=1$. Thus [11] there exists $m(t)$ increasing on $[0,2 \pi]$ with $\int_{0}^{2 \pi} d m(t)=1$, such that

$$
\frac{1}{1-p^{2}} P\left(\frac{z+p}{1+p z}\right)=\int_{0}^{2 \pi} \frac{1+e^{i t} z}{1-e^{i t} z} d m(t) .
$$

Thus

$$
P(z)=\left(1-p^{2}\right) \int_{0}^{2 \pi} \frac{(1-p z)+e^{i t}(z-p)}{(1-p z)-e^{i t}(z-p)} d m(t) .
$$

Expanding the integrand in powers of $z-p$ and integrating we obtain

$$
c_{1}=2 \int_{0}^{2 \pi} e^{i t} d m(t)
$$

and

$$
c_{2}=\frac{2}{1-p^{2}} \int_{0}^{2 \pi}\left(e^{2 i t}+p e^{i t}\right) d m(t)=\frac{2}{1-p^{2}} \int_{0}^{2 \pi} e^{2 i t} d m(t)+\frac{p c_{1}}{1-p^{2}} .
$$

Thus

$$
\left(1-p^{2}\right) c_{2}-p c_{1}-c_{1}^{2}=2 \int_{0}^{2 \pi} e^{2 i t} d m(t)-c_{1}^{2}
$$

Now let

$$
T(z)=\int_{0}^{2 \pi} \frac{1+e^{i t} z}{1-e^{i t} z} d m(t) .
$$

Then $\operatorname{Re} T(z)>0$ for $z \in \Delta$ and $T(0)=1$. If

$$
T(z)=1+p_{1} z+p_{2} z^{2}+\ldots, \quad z \in \Delta,
$$

then

$$
p_{1}=2 \int_{0}^{2 \pi} e^{i t} d m(t)=c_{1} \quad \text { and } \quad p_{2}=2 \int_{0}^{2 \pi} e^{2 i t} d m(t) .
$$

Thus from (5.9),

$$
\left(1-p^{2}\right) c_{2}-p c_{1}-c_{1}^{2}=p_{2}-p_{1}^{2} .
$$

But it is known [6] that $\left|p_{2}-p_{1}^{2}\right| \leq 2$. Thus

$$
\left|\left(1-p^{2}\right) c_{2}-p c_{1}-c_{1}^{2}\right| \leq 2 .
$$

Therefore from (5.8) we obtain

$$
2\left(1-p^{2}\right)^{2}\left|a_{1}\right| \leq 2\left|a_{-1}\right|,
$$

which is (5.5). 
Next, from (5.6),

$$
\left|a_{0}-w_{0}\right|=\frac{\left|a_{-1}\right|\left|c_{1}+p\right|}{1-p^{2}}=\frac{\left|a_{-1}\right|\left|p_{1}+p\right|}{1-p^{2}} \leq \frac{\left|a_{-1}\right|(2+p)}{1-p^{2}} .
$$

To see sharpness, consider

$$
f(z)=w_{0}+p w_{0} \frac{(1-z)^{2}}{(z-p)(1-p z)} .
$$

Since

$$
\frac{(z-p)(1-p z) f^{\prime}(z)}{f(z)-w_{0}}=-\left(1-p^{2}\right) \frac{1+z}{1-z},
$$

$f(z)$ is a member of $\Sigma^{\mathrm{s}}\left(p, w_{0}\right)$. Moreover, $\overline{\mathbb{C}} \backslash f[\Delta]$ is the line segment $\xi=t w_{0}$, $\left(1+p^{2}\right) /(1+p)^{2} \leq t \leq\left(1+p^{2}\right) /(1-p)^{2}$. Also, for $|z-p|<1-p$,

$$
\begin{aligned}
f(z)= & \frac{p w_{0}(1-p)}{(1+p)(z-p)} \\
& +\left[w_{0}+\frac{p\left(p-2+p^{2}\right)}{(1+p)\left(1-p^{2}\right)} w_{0}\right]+\frac{p w_{0}}{(1-p)(1+p)^{3}}(z-p)+\ldots,
\end{aligned}
$$

from which we can see that equality is attained in (5.4) and (5.5).

Theorem 9. With the notation of Theorem 8 ,

$$
\left|a_{-1}\right| \leq \frac{p(1-p)}{1+p}\left|w_{0}\right|
$$

and the inequality is sharp.

Proof. With $P(z)$ as in the proof of Theorem 8,

$$
\frac{d}{d z} \log (z-p)\left(f(z)-w_{0}\right)=\frac{(1-p z)-P(z)}{(z-p)(1-p z)} .
$$

Integrating, we obtain

$$
f(z)-w_{0}=\frac{p w_{0}}{z-p} \exp \int_{0}^{z} \frac{(1-p \xi)-P(\xi)}{(\xi-p)(1-p \xi)} d \xi .
$$

Thus

$$
a_{-1}=\lim _{z \rightarrow p}(z-p)\left(f(z)-w_{0}\right)=p w_{0} \exp \int_{0}^{p} \frac{(1-p \xi)-P(\xi)}{(\xi-p)(1-p \xi)} d \xi
$$

and

$$
\left|a_{-1}\right|=p\left|w_{0}\right| \exp \int_{0}^{p} \frac{(1-p \xi)-\operatorname{Re} P(\xi)}{(\xi-p)(1-p \xi)} d \xi .
$$

We can write

$$
P(z)=\left(1-p^{2}\right) Q\left(\frac{z-p}{1-p z}\right)
$$


where $\operatorname{Re} Q(z)>0$ for $z \in \Delta$ and $Q(0)=1$. Using the well-known inequality $\operatorname{Re} Q(z) \geq(1-|z|) /(1+|z|)$, we obtain for $\xi$ real and $0 \leq \xi \leq p$,

$$
\begin{aligned}
\operatorname{Re} P(\xi) & \geq\left(1-p^{2}\right) \frac{1-\left|\frac{\xi-p}{1-p \xi}\right|}{1+\left|\frac{\xi-p}{1-p \xi}\right|} \\
& =\left(1-p^{2}\right) \frac{1-\frac{p-\xi}{1-p \xi}}{1+\frac{p-\xi}{1-p \xi}}=(1-p)^{2} \frac{1+\xi}{1-\xi} .
\end{aligned}
$$

Combining (5.10) and (5.11) gives

$$
\begin{aligned}
\left|a_{-1}\right| & \leq p\left|w_{0}\right| \exp \int_{0}^{p} \frac{(1-p \xi)-(1-p)^{2} \frac{1+\xi}{1-\xi}}{(\xi-p)(1-p \xi)} d \xi \\
& =p\left|w_{0}\right| \exp \int_{0}^{p} \frac{p \xi+(p-2)}{(1-\xi)(1-p \xi)} d \xi \\
& =p\left|w_{0}\right| \exp \int_{0}^{p}\left(\frac{-2}{1-\xi}+\frac{p}{1-p \xi}\right) d \xi=p\left|w_{0}\right|\left(\frac{1-p}{1+p}\right),
\end{aligned}
$$

which is the inequality to be proven. Equality is attained by the function given in Theorem 8.

COROLlary. With notation of Theorem 8 ,

and

$$
\left|a_{0}-w_{0}\right| \leq \frac{p(p+2)}{(1+p)^{2}}\left|w_{0}\right|
$$

Both inequalities are sharp.

$$
\left|a_{1}\right| \leq \frac{p}{(1-p)(1+p)^{3}}\left|w_{0}\right|
$$

\section{References}

[1] L. de Branges, A proof of the Bieberbach conjecture, Acta Math. 154 (1985), 137-152.

[2] A. W. Goodman, Functions typically-real and meromorphic in the unit circle, Trans. Amer. Math. Soc. 8 (1950), 92-105.

[3] J. A. Jenkins, On a conjecture of Goodman concerning meromorphic univalent functions, Michigan Math. J. 9 (1962), 25-27.

[4] W. E. Kirwan and G. Schober, Extremal problems for meromorphic univalent functions, J. Analyse Math. 30 (1976), 330-348. 
[5] Y. Komatu, Note on the theory of conformal representation by meromorphic functions I, II, Proc. Japan Acad. 21 (1945), 269-284.

[6] A. E. Livingston, The coefficients of multivalent close to convex functions, Proc. Amer. Math. Soc. 21 (1969), 545-552.

[7] J. Miller, Convex meromorphic mappings and related functions, ibid. 25 (1970), 220-228

[8] -, Starlike meromorphic functions, ibid. 31 (1972), 446-452.

[9] -, Convex and starlike meromorphic functions, ibid. 80 (1980), 607-613.

[10] J. Pfaltzgraff and B. Pinchuk, A variational method for classes of meromorphic functions, J. Analyse Math. 24 (1971), 101-150.

[11] W. C. Royster, Convex meromorphic functions, in Mathematical Essays Dedicated to A. J. MacIntyre, Ohio Univ. Press, Athens, Ohio, 1970, 331-339.

[12] G. Schober, Univalent Functions-Selected Topics, Lecture Notes in Math. 478, Springer, Berlin, 1975.

DEPARTMENT OF MATHEMATICAL SCIENCES

UNIVERSITY OF DELAWARE

501 EWING HALL

NEWARK, DELAWARE 19716

U.S.A.

Reçu par la Rédaction le 4.10.1993 